\title{
Gone for good?
}

TODAY in Geneva, the World Health

Organisation will of ficially declare smallpox

eradicated from the Earth. It will be the culmination of an astonishingly successful

22-year programme by the WHO, which included, in the two years since the last endemic case was reported in Somalia, the unclaimed $\$ 1,000$ reward offered for new cases reported (below). But could smallpox ever recur? Peter Newmark examines the evidence.

THE WHO campaign to eradicate smallpox was launched in 1958, a year in which 250,000 cases of the disease were recorded. At first success was slow to come by. Even vaccination of $90 \%$ of the population of a country was sometimes found to leave a sufficient residuum of the virus for it to bounce back with a vengenance.

By 1967 the disease was still endemic, or in serious danger of being so, in 50 countries. The WHO therefore hatched and launched an intensified campaign of eradication.

By any reckoning, the success of that campaign was remarkable. Ten years and $\$ 300$ million later the last case of endemic smallpox was recorded.

Two and a half years later, the WHO is convinced that smallpox has gone for ever. In coming to that conclusion the WHO has considered three possible routes of reinfection but deemed them to be most unlikely, if not impossible.

The first would be if the virus could lie dormant in smallpox scabs that have survived this long. But the WHO is satisfied that the virus quickly becomes harmless in scabs.

The second possible source of reinfection comes from the smallpox virus stocks that are being held in the seven WHO-approved and inspected laboratories. That possibility last became a reality in Birmingham, UK, in 1978 when one worker died and another became mildly ill. Since these were not endemic cases they did not count within the WHO's definition of the 'last' cases. They did however, lead the WHO to increase its biosafety requirements and inspections, and to speed up its policy of closing down as many smallpox laboratories as possible. In two years, it is hoped, there will be only four, but some will have to be maintained indefinitely because of the possibility, however remote, that the virus could reappear from some other source, necessitating a new programme of diagnosis and vaccination. WHO plans to keep in reserve sufficient freeze-dried vaccine for 200 million people.

Meanwhile research is continuing on the

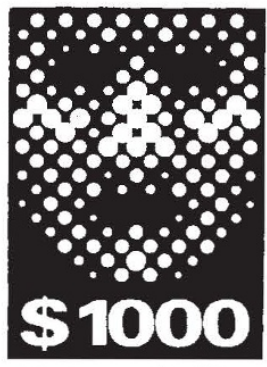

third conceivable route of reemergence, by mutation from monkeypox virus. That possibility was raised in 1978 by the publication in Nature of experiments carried out in the WHO-sponsored Research Institute of Virus Preparations in Moscow, under the direction of Dr S S Marennikova. These showed that both in the course of passage of monkeypox virus through hamsters, and in the pock appearing on chorioallantoic membranes infected with monkeypox, there sometimes emerged a virus that was indistinguishable from smallpox virus in laboratory tests.

That conclusion was controversial from the start because of the considerable differences between the smallpox and monkeypox virus; although both are members of the orthopoxvirus family, as is vaccinia virus from which smallpox vaccine is produced, monkeypox very rarely infects man and, when it does, appears not to be transmitted from person to person; in the laboratory every test, from its molecular characteristics to the colour of the pocks it produces, distinguishes it from smallpox. Whereas that does not preclude the possibility of mutation of monkeypox to smallpox virus, recent, as yet unpublished restriction enzyme analysis of the genome of twenty-one mutants newly derived from monkeypox show that, despite instances of major deletions and rearrangements of genes, all of the mutants were still recognizable as monkeypox virus, and none closely resembled smallpox virus. The laboratories responsible for this work (those of Prof. K R Dumbell, in St Mary's Hospital Medical School, London, and Dr J $\mathrm{H}$ Nakano at the Center for Disease Control, Atlanta, USA) have also analysed the genome of six of the mutants obtained from monkeypox in Moscow in 1978, and the two monkeypox stocks from which level, restriction enzyme maps of the two viruses show that the differences between them are so considerable that as Dr Nakano told Nature: "it seems highly improbable that the smallpox virus could ever be derived from the monkeypox virus by mutation".

However, Dr Nakano's laboratory has also analysed the four smallpox-like viruses isolated in Moscow from white pock on chorioallantoic membranes infected with monkeypox. Each of these mutants was indistinguishable from smallpox virus, confirming Dr Marennikova's identification of them.

The current position therefore seems to be that whereas it has not been possible to reproduce Dr. Marennikova's derivation of smallpox virus from monkeypox virus, and the genomic differences between the two viruses make such an event highly improbable, yet the evidence is clear that the Russian mutants are indistinguishable from smallpox.

There are two possible explanations, both unpalatable in their own way. The first is that Dr. Marennikova's results were the result of contamination. Obviously that can never be ruled out, and one of her two original monkeypox virus strains does appear to show signs of contamination with smallpox virus; however, the other does not.

The other possible explanation, and much the more worrying one, is that, despite the improbability, monkeypox virus can mutate to smallpox virus. That possibility is described by WHO as "highly unlikely, but impossible to disprove". All it can do is to continue to sponsor research - and hope that today's declaration of smallpox eradication is the very last one. $\square$ 